\title{
Load Forecasting Using Back Propagation Algorithm
}

\author{
Manish Kumar Singla, Jyoti Gupta
}

(Electrical and Instrumentation Engineering Department, Thapar Institute of Engineering and Technology

Patiala, India.)

\section{Abstract:}

The key role of load forecasting is the power system energy management system. Load forecasting helps to diminish the production cost, spinning reserve capacity and enhance the reliability of the power system. Load forecasting is tremendously essential for financial institutions, power suppliers and other participants in electric energy market i.e. transmission, generation and distribution. The economic allotment of generation is a vital purpose of short term load forecasting. This paper presents a solution methodology using an artificial neural network for short term load forecasting. The inputs using for forecasting the load, i.e. Dry bulb temperature, Dew point temperature, humidity and load data. The load data is taken from the $66 \mathrm{kv}$ substation, Bhai Roopa, Bathinda and weather data from weather stations "IMD" Pune. The data are taken from the year 2015 and 2016. The back propagation algorithm has been implemented to minimize the error function derived on the basis of computed load and actual load. The effectiveness is also checked through its implemented under the MATLAB environment.

Keywords - component; Short term load forecasting, Back Propagation, Temperature, Humidity, Neural Network.

\section{INTRODUCTION}

Power utilities are needed to supply reliable power to consumers. Within style stages, utilities have to be compelled to set up ahead for predictable future load development underneath totally different attainable situations. Their choices and styles will have an effect on the profit or loss of crores of rupees for his or her organizations/utilities moreover as client fulfillment and future monetary process in their space. For efficient operation and planning of utility company, correct models of power load prediction are necessary. Load forecasting is a very essential tool for an electrical utility to form necessary choices together with choices on the purchase, also for banking of power (with alternative corporations or identical state utilities or with the neighboring states), in the generation of power, in load change and development in infrastructure [1]. It is very necessary for energy suppliers as well as for other alternative participants within the electrical energy transmission, generation, distribution, and markets.

For electric utilities, accurate forecasting of load accurately plays an awfully necessary role due to exceeding cutthroat competitive surroundings shaped by the electric business deregulating. An electric company is confronted with several economists and technical operational issues, along with planning and management of a utility electric system since customers should be provided electricity of highquality in an exceedingly efficient and safe manner [1]. Load forecasting is also beneficial for an electric utility in creating necessary selections on generating, interchanging, and buying wattage, load change. Besides this, it is important for suppliers' utility, many establishments and others concerned with the electric energy generation and regulation [2].

Load forecasting strategies can be classified into four parts i.e. very short, short, medium and long-term models in accordance with the time duration. In veryshort-term forecasting, the time span may be short a few minutes whereas in long-term forecasting it may start with few years and can extend up to few decades. In dissertation work, short-term load forecasting is mainly concentrated.

With the modern phenomena of the liberation of electricity markets, STLF has acquired additional importance and larger problems. Within the atmosphere of the market, precise forecasting is that foundation of the trading of power and the damage establishment of the system to achieve the lowest electricity buying price. 


\section{SHORT TERM LOAD FORECASTING}

\section{A. Background}

In power system planning, generation and transmission, operation and control the load forecasting plays a crucial part [3]. Forecasting suggests that calculating active load at numerous load buses previous actual load prevalence. Application of load forecasting in planning and operation needs an exact 'lead time' additionally known as forecasting intervals. Categorization of load forecasting with respect to lead time is presented in Table 1 .

Table 1 Categorization of Load Forecasting.

\begin{tabular}{|c|c|c|}
\hline $\begin{array}{c}\text { Nature of } \\
\text { forecast }\end{array}$ & Lead time & Applications \\
\hline Very short term & $\begin{array}{c}\text { few seconds to } \\
\text { few minutes }\end{array}$ & $\begin{array}{c}\text { Scheduling of generation } \\
\text { and distribution, power } \\
\text { system security analysis }\end{array}$ \\
\hline Short term & $\begin{array}{c}\text { Half an hour to the } \\
\text { number of hours }\end{array}$ & $\begin{array}{c}\text { Unit commitment and } \\
\text { spinning reserve } \\
\text { allocation }\end{array}$ \\
\hline Medium term & $\begin{array}{c}\text { Few days to a } \\
\text { number of weeks }\end{array}$ & $\begin{array}{c}\text { Planning for seasonal } \\
\text { peak winter, summer }\end{array}$ \\
\hline Long-term & Up to one year & $\begin{array}{c}\text { Planning generation } \\
\text { growth. }\end{array}$ \\
\hline
\end{tabular}

There are mainly three types of categorization for load: short-term forecasting generally done from few hours to one week, medium-term forecasting is done from few weeks to a year, and long-term forecasting is done for more than one year. In an organization, it is necessary to forecast at various time horizon for various operations. These forecasts are distinct in nature. Most of the strategies employ statistical methods or artificial intelligence algorithms like fuzzy logic, regression, expert system and neural networks. From medium and long-term load forecasting, enduse econometric technique is widely used. For STLF, various strategies are employed such as fuzzy logic, different regression models, statistical learning techniques, time series, expert systems and similar day methods.

\section{B. Necessary Factors For Forecasting}

For short-term load prediction, similar to the time factors, weather data, and potential customer categories, many variables should be considered. Medium and long-term forecasts, chronological weight and weather data, consider the number of customers in the Different categories, areas within the field and their characteristics, as well as age, economic and demographic data, and their predictions, equipment sales data, and various Factors forecasts, the appliance sales data, and different factors.

Weather conditions influence the load. In fact, a predicted weather parameter is the most important factor in short-term load forecasts. Many weather variables can be thought of as load forecast. Temperature and Humidity are the most commonly used load predictions. Most electric utilities serve clients of $\mathrm{di} \square$ erent sorts, such as commercial, industrial, and residential. The power usage pattern is for di $\square$ erent customers which are related to di $\square$ erent categories, however, in some categories, it is somewhat similar to the customers.

\section{Forecasting Methodology}

Forecasting techniques can be divided into three broad categories. These include extrapolation, correlation, and the combination of both the techniques. Techniques can be subclassified as deterministic, probabilistic or stochastic.

(i) Extrapolation involves fitting trend curves to basic chronological data adjusted to replicate the growing trend itself [4]. The most common curve fitting technique for fitting coefficients and exponents of a function in a very given forecast is that the methodology of least squares.

(ii) Correlation method based technique of forecasting relates system loads to numerous demographic and economic factors. This approach is advantageous in forcing the forecaster to know clearly the interrelation between load growth patterns and different measurable factors.

\section{Classification Of Load Forecasting Methods}

Load forecasting strategies are categorized into four classes [3] in terms of lead time.

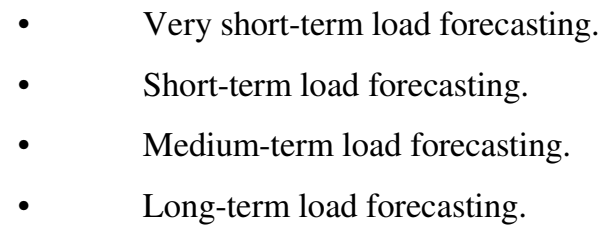

Short-term load forecasting can use to evaluate load flows and also helpful in prohibiting overloading. Aforementioned selections prompt network reliability and decrease happening of apparatus failures and power outages. Within the deregulated economy, selections on costs supported long-term forecasting also are additional necessary than in a very nonderegulated economy once rate will increase may well be even by capital expenditure projects. 
For short-term load forecasting, numerous variables must be deliberated, similar to climate data, time factors, and possible consumer's categories whereas the medium and long-term forecasts consider the climate data and historical data, number of on the various weekdays can change in a different manner, for instance, Mondays and Fridays being nearby ends of the week, may have basically unexpected loads in comparison to Tuesday through Thursday. This is especially valid amid the late spring. Holidays are more crucial to forecast as compared to working days in view of their relatively rare incidence. The difference in the climate causes, the difference in the customer's amenity feeling and thus utilization several apparatus corresponding to the refrigerator, water heater, tv.

\section{BACKPROPAGATION ALGORITHM}

\section{A. Overview}

Back Propagation (BP) alludes to a wide group of ANN, whose design comprises of various interconnected layers. The learning algorithm of BP is based on the Deepest Descent technique. The proper number of hidden units limits the error of the non-linear function of high complexity. BP is a systematic technique of training multilayer ANN. It is designed on a high mathematical foundation and has excellent application potential [5]. The networks include sensory units that represent the input layer, one or more hidden layers of calculation nodes, and the output layer of the calculation nodes. Input signals on a layer-by-layer basis propagate through the network in the forward direction. These neural networks normally allude to as a multilayer perceptrons as shown in Figure 1.

\section{B. Algorithm}

The BP algorithm involves four stages and flow chart gives full explanation of algorithm the flow chart is shown in Figure 2.

1. Initialization of weights: Some small random values are assigned.

2. Feed forward: Each input unit $(\mathrm{X})$ receives an input signal and transmits this signal to each of the hidden units. Each hidden units then calculates the activation function and sends its signal to each output unit. The output unit calculates the activation function to form the response of the given input pattern.

3. Back propagation error: Each output unit compares activation with its target value to determine the associated error for that unit. Based on the error, the factor is computed and is used to distribute the customers in numerous categories, the apparatuses in the zone, economic and statistic data and their forecast, the apparatus deals information and different factors. There are necessary fluctuations in load amongst the weekdays and end of the week. The load error at output unit back to all units in the previous layer. Similarly the factor is compared for each hidden units.

4. Updation of the weights and biases

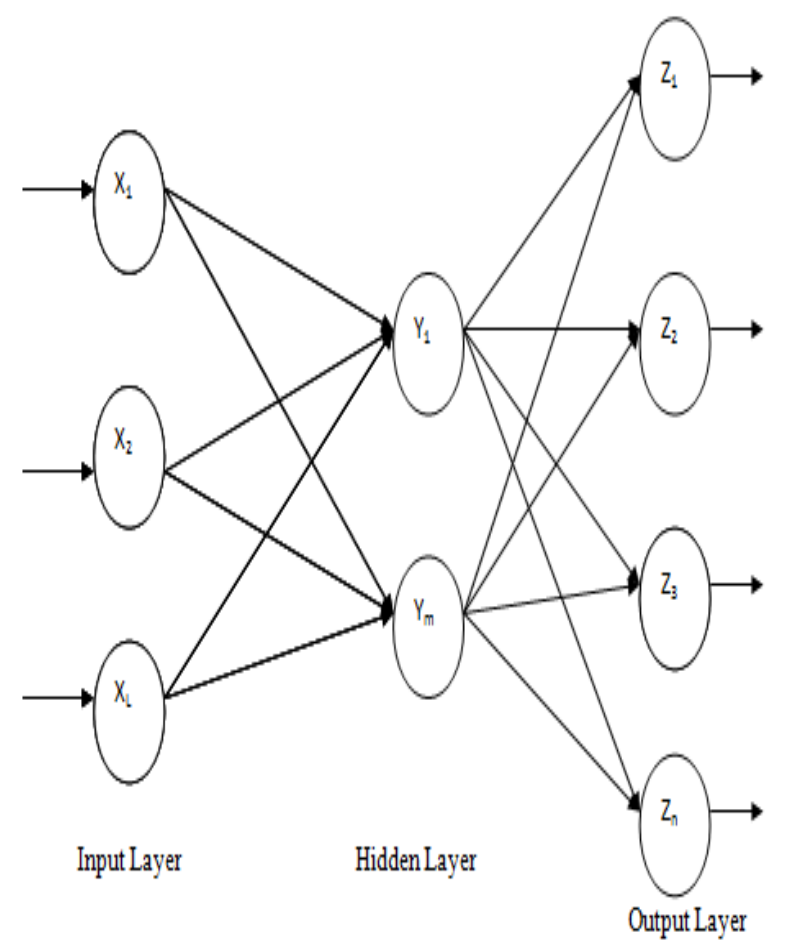

Figure 1 Multi-Layer Fed Forward Network.

\section{Results and Discussions}

Back propagation was considered as the important ingredient in the short term forecasting. For the forecasting purpose, hourly data was collected from the Punjab electricity board for the various days that helped in the training of the technique as well as for forecasting purpose and also the weather data is taken from the IMD Pune. The dew point temperature, dry bulb temperature, and humidity are taken as input and the load data is taken as the output. To train the network data, the data are divided into three parts, i.e. Validation, Training, and Testing. The BP algorithm is used to train the network and implemented in MATLAB. The inputs dew point, dry bulb temperature, humidity and load are presented in Figure.3

(i) Dew Point Temperature: It was basically a 
temperature value at which the air losses its control over the water vapor due to which some of the air molecules converted in to the water droplet and this particular temperature was lower than the temperature.

(ii) Dry Bulb Temperature: whenever the thermometer was used for the measurement of temperature then it is called dry bulb temperature basically, it was atmospheric temperature read by the thermometer whenever it was exposed in the surrounding.

(iii) Humidity: it was nothing but a water droplet which was present the atmosphere but they were completely invisible for the human was basically a water molecules in the gaseous state. As humidity -

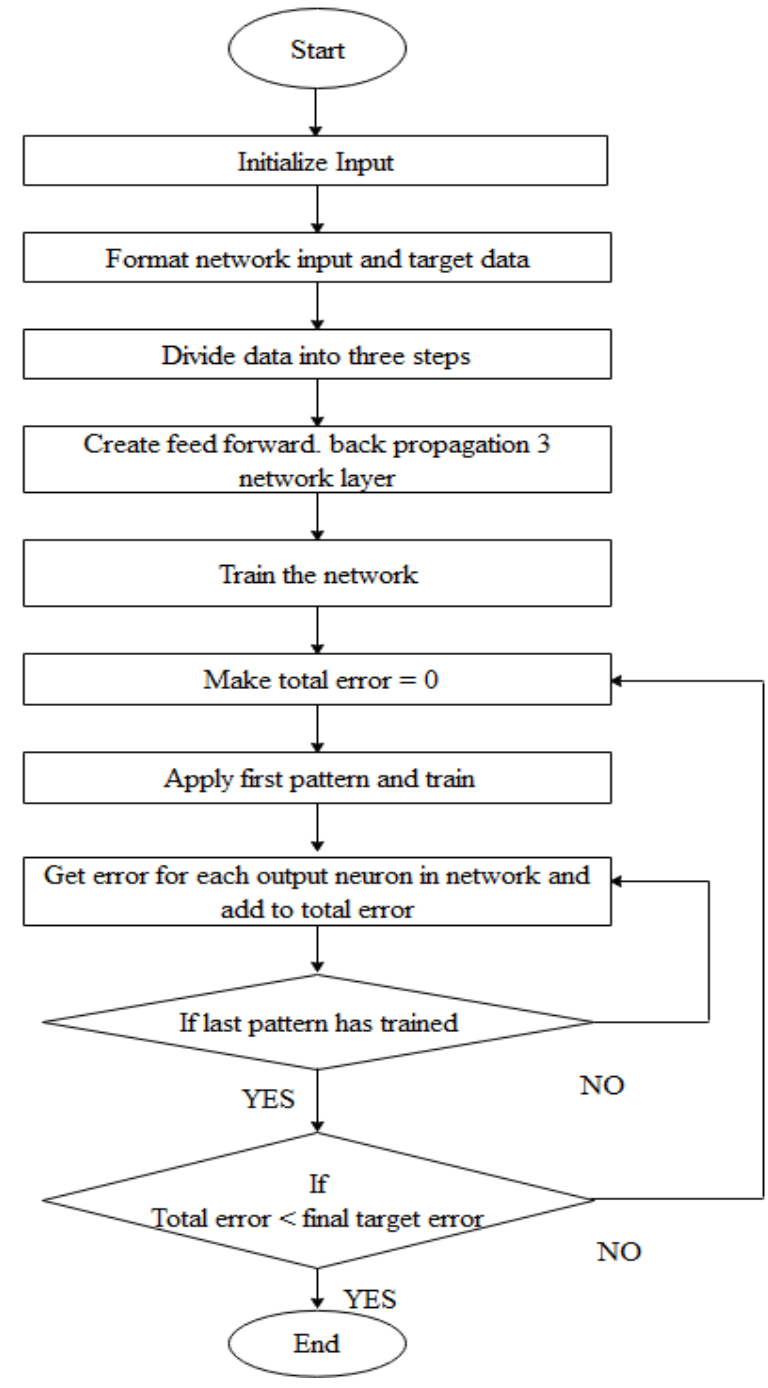

Figure 2 Back propagation flow chart increases, ability of body to resist the sweating capacity reduces due to reduction in the rate of evaporation of moisture from the body.

The network is trained for 5000 iterations with the presented data. In this, the training function is 'trainlm' and the activation function is sigmoid. It is observed that the mean square error is 0.0061 and root mean square error is 0.0784 after training it is also observed that the actual load and forecasted load have so much variation or fluctuations as shown in Fig. 4. As there is a large variation between actual and forecasted load obtained by BP algorithm.

The short-term load forecasting is performed with the help of BP algorithm. Initially, the data for January month has been used in the normalized form. The updated values of weights have been obtained by training of network. The values of the initial and the final values of the weights between input and hidden layer are presented in Table 2. Table 3 presents the initial and final weights between hidden and an output layer. The size of neurons for input layer and hidden layer is selected as 3 and 21 respectively. The weights are initialized randomly.

\section{ConCLUSION}

In this paper the author discuss about the short term load using the back propagation algorithm. The input data is collected from the weather station and load data is collected from the load station. In this paper the MSE and RMSE error is calculated. It is concluded that the MSE error is 0.0061 and RMSE error is 0.0784 respectively. The network is trained with the 5000 iterations and the training function used is "trainlm". The weights are calculated with the help of back propagation code. For the load forecasting neural network using back propagation algorithm is the best suited algorithm to calculate the error. 

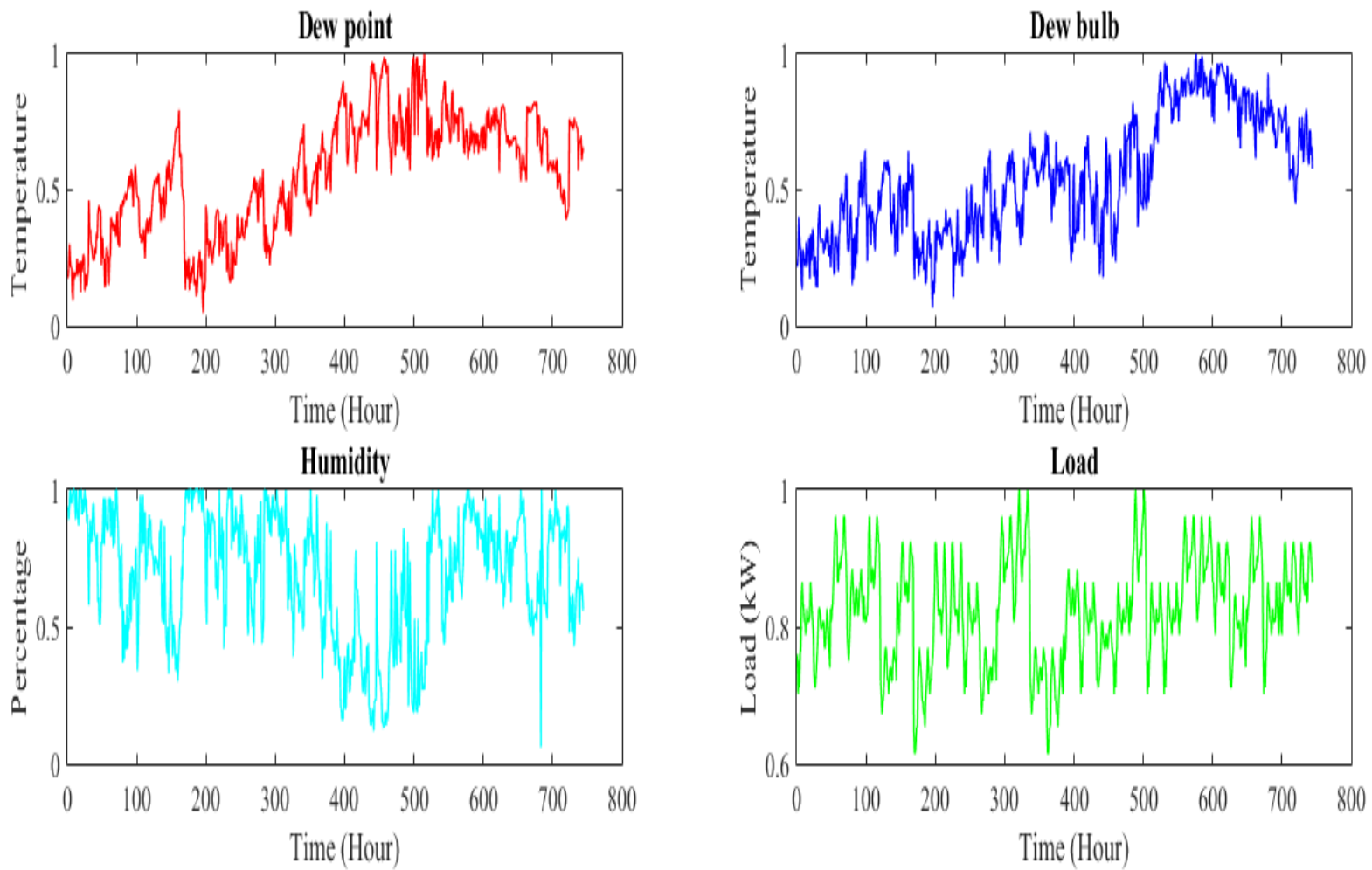

Figure 3 Input data representation

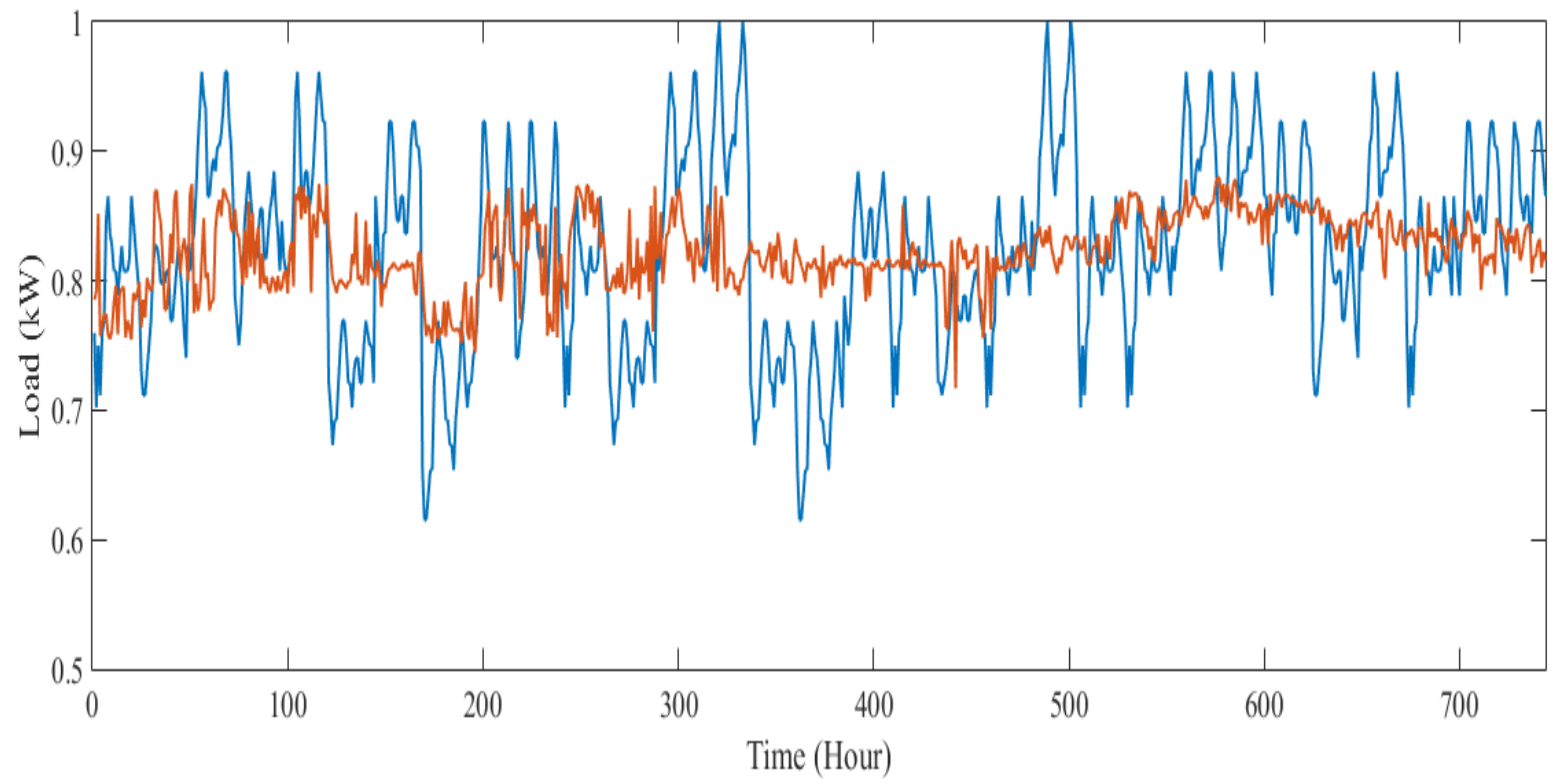

Figure 4 Actual and Forecasted Load Graph 
Table 2 Initial and final weights between input and hidden layer

\begin{tabular}{|c|c|c|c|c|c|c|c|c|}
\hline \multirow{2}{*}{$\begin{array}{c}\text { Input } \\
\text { layer } \\
\text { Hidden } \\
\text { Layer }\end{array}$} & \multicolumn{2}{|c|}{1} & \multicolumn{2}{|c|}{2} & \multicolumn{2}{|c|}{3} & \multicolumn{2}{|c|}{4} \\
\hline & $\begin{array}{l}\text { Initial } \\
\text { weight }\end{array}$ & $\begin{array}{c}\text { Final } \\
\text { Weight }\end{array}$ & $\begin{array}{l}\text { Initial } \\
\text { weight }\end{array}$ & $\begin{array}{c}\text { Final } \\
\text { Weight }\end{array}$ & $\begin{array}{l}\text { Initial } \\
\text { weight }\end{array}$ & $\begin{array}{c}\text { Final } \\
\text { Weight }\end{array}$ & $\begin{array}{l}\text { Initial } \\
\text { weight }\end{array}$ & $\begin{array}{c}\text { Final } \\
\text { Weight }\end{array}$ \\
\hline 1 & -0.34782 & -1.80347 & -0.43868 & 0.47153 & 0.405632 & -0.02584 & 0.2499 & 1.263513 \\
\hline 2 & 0.123935 & 0.289727 & -0.31757 & 1.177436 & 0.200417 & 1.60001 & -0.10008 & -0.99536 \\
\hline 3 & -0.22342 & -0.47633 & -0.22485 & 0.212142 & 0.032243 & 0.102828 & 0.199506 & 0.149904 \\
\hline 4 & -0.48403 & -4.87617 & 0.453785 & 9.75576 & 0.119968 & 15.20919 & -0.41221 & -6.54275 \\
\hline 5 & 0.473032 & 0.825447 & -0.34053 & -1.09561 & -0.38254 & -0.31897 & 0.163085 & -0.21496 \\
\hline 6 & -0.38572 & -0.4897 & -0.14099 & -1.72231 & -0.36486 & -0.70368 & -0.11197 & 0.995499 \\
\hline 7 & -0.27127 & -0.22165 & -0.16856 & -0.00151 & -0.08398 & 0.184493 & -0.06536 & 0.093757 \\
\hline 8 & -0.38854 & -2.30037 & 0.321911 & 0.745717 & 0.21104 & -2.28622 & 0.294604 & 4.21837 \\
\hline 9 & 0.473491 & 1.200004 & -0.34527 & -0.32894 & 0.210566 & 0.439223 & -0.1994 & -0.2442 \\
\hline 10 & -0.34622 & -0.58212 & 0.030134 & 1.072627 & 0.109414 & 0.507845 & -0.11479 & 0.000284 \\
\hline 11 & 0.194902 & 0.107761 & -0.11681 & 0.010768 & 0.25956 & 0.121974 & 0.378944 & 0.306346 \\
\hline 12 & -0.37715 & -0.0205 & 0.441032 & 1.010085 & 0.084463 & 0.80182 & 0.404168 & 1.499915 \\
\hline 13 & 0.218026 & 0.175655 & 0.451569 & 0.89646 & -0.10817 & -0.24252 & 0.285362 & -0.13567 \\
\hline 14 & 0.407962 & 0.463122 & 0.494323 & 1.611107 & 0.493919 & 0.628435 & -0.19042 & -0.81396 \\
\hline 15 & 0.238622 & -5.49304 & -0.12286 & 9.250211 & 0.106339 & 9.479236 & -0.4836 & -2.02213 \\
\hline 16 & -0.26786 & -0.81224 & -0.11897 & -1.98228 & 0.427006 & 0.895768 & -0.33477 & 0.684218 \\
\hline 17 & -0.28366 & -0.17115 & 0.169387 & -0.85874 & 0.376344 & 5.69967 & -0.24377 & -0.81127 \\
\hline 18 & 0.216537 & 0.29322 & -0.37409 & -0.74481 & -0.41176 & -0.17854 & -0.02559 & 0.147388 \\
\hline 19 & 0.287573 & 0.726257 & -0.08337 & -0.13256 & -0.21717 & 0.189039 & -0.11786 & 0.034249 \\
\hline 20 & -0.09315 & 4.299436 & -0.37366 & -5.59736 & -0.44577 & -4.29019 & -0.46507 & -1.38879 \\
\hline 21 & 0.309573 & -0.02732 & 0.008597 & -1.77245 & -0.34976 & -0.89054 & 0.494925 & 0.765769 \\
\hline
\end{tabular}


Table 3 Initial and final weights between hidden and output layer

\begin{tabular}{|c|c|c|}
\hline \multirow{2}{*}{$\underbrace{}_{\substack{\text { Input } \\
\text { Lay } \\
\text { Layer }}}$} & \multicolumn{2}{|l|}{1} \\
\hline & $\begin{array}{l}\text { Initial } \\
\text { weight }\end{array}$ & $\begin{array}{c}\text { Final } \\
\text { Weight }\end{array}$ \\
\hline 1 & -0.40842 & -1.46514 \\
\hline 2 & 0.343397 & 0.752626 \\
\hline 3 & 0.4595 & 1.455527 \\
\hline 4 & 0.349397 & -0.06603 \\
\hline 5 & -0.35115 & -4.90491 \\
\hline 6 & -0.32571 & -1.71731 \\
\hline 7 & -0.14839 & -1.81978 \\
\hline 8 & 0.181918 & -0.47673 \\
\hline 9 & 0.44912 & 3.267875 \\
\hline 10 & -0.36074 & -1.58596 \\
\hline 11 & 0.453228 & 0.562534 \\
\hline 12 & 0.327723 & -0.46041 \\
\hline 13 & -0.34338 & -1.77566 \\
\hline 14 & 0.078922 & 0.182285 \\
\hline 15 & -0.45145 & 1.054586 \\
\hline 16 & 0.493923 & 7.432081 \\
\hline 17 & -0.2786 & -1.76668 \\
\hline 18 & 0.404807 & 3.120187 \\
\hline 19 & 0.157243 & -1.12301 \\
\hline 20 & -0.10462 & -1.18771 \\
\hline 21 & -0.08841 & 6.37731 \\
\hline
\end{tabular}

\section{References}

[1] Singla, M., 2018. Load Forecasting Using Artificial Neural Network (Doctoral dissertation).

[2] Manish Kumar Singla, Thapar Institute of Engineering and Technology, Patiala; Sikander Hans ,Thapar Institute of Engineering and Technology, Patiala. "Load Forecasting using Fuzzy Logic Tool Box." Global Research and Development Journal For Engineering 38 2018: $12-19$.

[3] Kothari, D. P., \&Nagrath, I. J. "Modern power system analysis". Tata McGraw-Hill Education, 2011.

[4] Hippert, Henrique Steinherz, Carlos Eduardo Pedreira, and Reinaldo Castro Souza. "Neural networks for short-term load forecasting: A review and evaluation". IEEE Transactions on power systems, vol. 16, no. 1, pp.44-55, 2001.

[5] Zurada, Jacek M."Introduction to artificial neural systems”. vol. 8. St. Paul: West, 1992.

[6] IMD Pune Weather Data. 